\title{
LIBERTAD DE CONCIENCIA EN EL CURRÍCULO OCULTO DE LA FACULTAD DE ENFERMERÍA
}

Olga Muñoz de Bermúdez*

\section{Resumen}

El objeto de la investigación es integrar en el currículo oculto de la facultad de enfermería de la Fundación Universitaria de Ciencias de la Salud (FUCS), la libertad de conciencia como una herramienta ética del enfermero para resolver dilemas éticos. Con este fin fue llevado a cabo un estudio descriptivo para saber si el enfermero vivía dilemas éticos y conocía la libertad de conciencia, mediante la aplicación de una encuesta semiestructurada a profesores, alumnos y egresados de la facultad de enfermería; de este modo pudo concluirse que el enfermero vive dilemas éticos con frecuencia y no conoce la libertad de conciencia, por lo que no aplica la objeción de conciencia en el desempeño de su labor profesional.

En consecuencia, se propone formar desde el pregrado al profesional de enfermería en la libertad de conciencia, de manera que esté en capacidad de rechazar situaciones que vayan en contra de su formación moral y profesional. Así, el enfermero mejorará su entorno laboral y logrará proteger los derechos del paciente, fundamentada en los derechos humanos y en la Constitución Política de Colombia.

\section{Introducción}

La libertad de conciencia es la libertad de seguir el dictamen de la razón sobre la moralidad de nuestro actuar; estamos obligados a seguir nuestra conciencia, así no sea la verdadera.

De la libertad de conciencia salen dos derechos: repudiar y objetar. Repudiación es rechazar, en nombre de la conciencia, el ejercicio de unos derechos, mientras que se realiza una objeción cuando se rechaza el cumplimiento de ciertos deberes. Es decir, en nombre de la conciencia se pueden repudiar derechos u objetar deberes.

La primera documentación escrita sobre la libertad de conciencia está en el Derecho Constitucional (1776) y más tarde en la asamblea Nacional de Francia (1789) y en las constituciones Latinoamericanas del siglo XIX. En 1961 surge la Fundación Amnistía Internacional, que defiende y promueve los derechos humanos, apoya la libertad de conciencia y expresión.

Por su parte, la Constitución Política de Colombia, en el artículo 18, habla de la libertad de conciencia como un derecho fundamental y hasta el momento se ha aplicado la libertad de conciencia en la religión, el servicio militar y la salud. En el área de enfermería sólo se encuentra aplicada en unos casos de Estados Unidos. En

* Facultad de Enfermería, Fundación Universitaria de Ciencias de la Salud, Bogotá, Colombia
Colombia solamente ha tenido aplicación en el servicio militar y la religión con los testigos de Jehová.

El enfermero es un profesional de la salud cuyo objetivo es el cuidado de enfermería y lo realiza previniendo, recuperando o rehabilitando a la persona que tiene alterado su estado de salud.

Actualmente el enfermero vive situaciones difíciles en su trabajo que le ocasionan dilemas éticos, como son: exceso de pacientes, insuficiente recurso humano y material, rotación de servicios, conductas inapropiadas de otros profesionales, exigencias del paciente y los familiares.

A través del trabajo se comprobó que el enfermero desconoce la libertad de conciencia y su utilidad para resolver los conflictos de orden ético dentro de su desempeño profesional. Para el análisis anterior se realizó una encuesta a docentes, alumnos y egresados, teniendo como temáticas, el cuidado de enfermería, los dilemas éticos, la conciencia, los derechos humanos y la libertad de conciencia.

\section{Materiales y métodos}

Al diseñar el método del objeto de investigación se trabajó una metodología de tipo cualitativo. La población seleccionada fueron los profesores, alumnos y profesionales egresados de la facultad de enfermería de la Fundación Universitaria de Ciencias de la Salud. Para seleccionar la muestra se trabajó con un índice de 
confiabilidad de $90 \%$. Los estudiantes seleccionados fueron los del octavo semestre, por considerar que este grupo ya ha recibido todos los elementos éticos del currículo oculto de la Facultad. Además, fueron escogidos los egresados que laboran en el Hospital de San José, por ser la institución que alberga mayor numero de ellos.

El tipo de investigación es descriptivo exploratorio, ya que indaga sobre la libertad de conciencia en la población de enfermería y describe la manera en que este grupo de profesionales la vivencia y aplica en su desempeño laboral.

El estudio busca describir los conocimientos sobre la libertad de conciencia en la población de enfermería, tomando como punto de partida su formación ética.

La enfermería es una profesión de servicio social, basada en la aplicación de principios científicos y éticos que contribuyen a promover, mantener y recuperar la salud del individuo, la familia y la comunidad; por lo tanto, requiere una investigación cualitativa que enfoca las ciencias sociales.

Los estudios cualitativos son de gran importancia para los profesionales de salud pues se centran en el cuidado, la comunicación y la interacción con las personas; también permiten una comprensión de las experiencias humanas, proporcionando a los profesionales de la enfermería un conocimiento de gran riqueza y profundización en la naturaleza de los seres humanos, ya sean pacientes o colegas.

Se tomó una muestra aleatoria con un nivel de confiabilidad de $95 \%$ y 0,5 de grado de error, que estuvo representada por 40 estudiantes de último semestre, 14 docentes de planta y 52 egresados, vinculados laboralmente al Hospital de San José, en la ciudad de Santa Fe de Bogotá, Colombia.

Teniendo como base la libertad de conciencia como aspecto ético de los profesionales de enfermería para resolver dilemas éticos, al elaborar el diagnóstico se buscaron las bases éticas del actuar de enfermería. Para ello se tuvo en cuenta el fundamento del cuidado de enfermería, el concepto de conciencia, la vivencia de dilemas éticos, la solución de los mismos, el conocimiento de derechos humanos y la aplicación de la libertad de conciencia y, por ende, de la objeción de conciencia.

En el cuidado de enfermería se tuvo en cuenta la prioridad del aspecto humano con respecto al profesional. En el aspecto humano fueron analizados el trato individual y el respeto, mientras que en el aspecto profesional se observó si se aplica la beneficencia y la justicia como principios éticos. En la conciencia se buscó el conoci- miento del enfermero sobre la formación y aplicación de este principio moral.

Ante la vivencia de dilemas éticos se quiso saber si se presentan y con quién ocurren más frecuentemente: pacientes, colegas, familia u otros profesionales. Ante las decisiones éticas se averiguó si el enfermero toma o no decisiones éticas y si lo hace por intuición o como resultado de su formación académica.

\section{Resultados}

El análisis de los resultados de las encuestas mostró la frecuencia con la cual se vivencian dilemas éticos, lo que permite deducir que el enfermero vive situaciones de conflicto permanentemente, independientes del campo de acción, es decir, ya sea como estudiante, como profesional egresado en ejercicio o como docente (Tabla 1).

Tabla 1

\begin{tabular}{|c|c|c|c|}
\hline \multicolumn{4}{|c|}{ Existencia de dilemas éticos } \\
\hline & Alumnos (\%) & Egresados (\%) & Profesores (\%) \\
\hline Nunca & 2 & 2 & 0 \\
\hline Raras vece & 10 & 17 & 11 \\
\hline Frecuente & 75 & 60 & 84 \\
\hline Siempre & 13 & 19 & 5 \\
\hline No respond & 0 & 2 & 0 \\
\hline
\end{tabular}

Al establecer con quién ocurren los dilemas éticos se observa que en el caso de los alumnos egresados y profesores predominaron con el paciente.

Lo anterior resulta claro al considerar que el paciente es el objeto central de atención del profesional de enfermería y puesto que dicho profesional está en permanente contacto con el enfermo, ello facilita el surgimiento de situaciones de conflicto o que por sus características son generadoras de conflictos éticos.

El enfermero vive en interacción permanente con otros profesionales de la salud y como coordinadora del equipo de atención de los pacientes interviene y toma decisiones que facilitan, en determinado momento, el desarrollo de cuestionamientos (Tabla 2).

\section{Tabla 2}

Distribución porcentual de los dilemas éticos y con quien se presentan

\begin{tabular}{|lccc|}
\hline & Alumnos (\%) & Egresados (\%) & Profesores (\%) \\
Pacientes & 55 & 41 & 59 \\
Colegas & 12 & 4 & 21 \\
Médicos & 13 & 33 & 5 \\
Familia & 10 & 18 & 5 \\
No responde & 10 & 4 & 10 \\
\hline
\end{tabular}


Al comparar los tres grupos de la muestra, se observa que al considerar separadamente los distintos factores involucrados en la toma de decisiones éticas, el mayor porcentaje es atribuido, en primer término, a los aspectos legales y luego, en orden de importancia, a los aspectos filosóficos y morales.

Esto demuestra que los enfermeros, en general, conocen los aspectos de una decisión ética y contradice el concepto según el cual los profesionales de enfermería tienen una confusión de lo ético, con lo legal (Tabla 3).

Tabla 3

\begin{tabular}{|lccc|}
\hline \multicolumn{4}{|c|}{ Aspectos de la decisión ética } \\
\hline & Alumnos (\%) & Egresados (\%) & Profesores (\%) \\
Legales & 0 & 4 & 0 \\
Filosóficos & 0 & 0 & 0 \\
Morales & 3 & 6 & 5 \\
Todas las & & 88 & 95 \\
anteriores & 97 & 2 & 0 \\
No responde & 0 & & \\
\hline
\end{tabular}

Todos los grupos encuestados asignan el mayor porcentaje para realizar objeción de conciencia al rechazo de un derecho, lo que demuestra la confusión que existe actualmente en la población de enfermería sobre el quehacer deontológico, en lo concerniente a los derechos y deberes (Tabla 4).

\section{Tabla 4}

\begin{tabular}{|c|c|c|c|}
\hline \multicolumn{4}{|c|}{ Hay objeción de conciencia cuando se rechaza } \\
\hline & Alumnos (\%) & Egresados (\%) & Profesores (\%) \\
\hline Derecho & 35 & 68 & 47 \\
\hline Idea & 27 & 6 & 16 \\
\hline Deber & 13 & 10 & 11 \\
\hline Acción & 20 & 6 & 26 \\
\hline No responde & e 5 & 10 & 0 \\
\hline
\end{tabular}

Llama la atención que en los tres grupos estudiados dentro de la muestra, un marcado porcentaje no ha aplicado la objeción de conciencia (Tabla 5).

\section{Tabla 5}

\begin{tabular}{|lccc|}
\hline \multicolumn{4}{|c|}{ Ejemplo de la objeción de conciencia } \\
\hline & Alumnos (\%) & Egresados (\%) & Profesores (\%) \\
No & 98 & 90 & 74 \\
Sí & 2 & 10 & 26 \\
& & & \\
\hline
\end{tabular}

La realidad es que los enfermeros no conocen sobre el tema y por esto no lo pueden aplicar en su práctica profesional.

Puesto que es válido aceptar el concepto de objeción de conciencia como un elemento moderno y ya que el enfermero vive situaciones de conflicto, es indispensable que los profesionales de enfermería se preparen éticamente para resolver situaciones que con frecuencia deben cumplir y que son incompatibles con su formación moral.

El enfermero es consciente del predominio de la humanización en el cuidado de enfermería, pero el ambiente laboral la obliga a actuar de manera diferente.

Los estudiantes egresados y profesores viven con frecuencia dilemas éticos y los diferencian de situaciones administrativas, legales y personales; por otra parte, la mayoría de los dilemas éticos ocurren con los pacientes.

Los datos recopilados muestran cómo el profesional de enfermería se forma con una actitud de sumisión y aceptación a situaciones contrarias a su conciencia, no conoce la libertad de conciencia y, por ende, no ejerce la objeción de conciencia.

\section{Discusión}

En la actualidad los profesionales de enfermería viven situaciones conflictivas con el paciente, los colegas y el equipo de salud. También encuentran circunstancias no apropiadas en su entorno laboral que les impiden realizar eficazmente su ejercicio profesional.

El enfermero desconoce la libertad de conciencia que le permitiría hacer objeciones de conciencia y, con ello, resolver los dilemas éticos que se le presentan con frecuencia.

El currículo oculto forma al estudiante con responsabilidad, le hace reconocer sus derechos y deberes con la ciencia y la sociedad y le brinda principios para enfrentar sus compromisos con su profesión y consigo mismo.

En estas condiciones, resulta claro que se debe crear un currículo oculto de la libertad de conciencia en la facultad de enfermería, de manera que el estudiante esté en capacidad de valorar la calidad moral de sus actos y discernir si lo que hace está de acuerdo a sus preceptos racionales, lo que le permitirá formar una norma interiorizada de moralidad.

La libertad de conciencia le permitirá a los profesionales de enfermería prepararse para afrontar los dilemas éticos a los que usualmente se ven enfrentados.

De esta manera, podría hacer objeciones de conciencia que vayan en contra de su formación profesional, lo cual también logrará optimar la calidad del cuidado de 
enfermería y mejorar el entorno laboral del profesional de enfermería.

\section{Conclusiones}

En la ultima década los profesionales de enfermería han recibido en la Universidad formación ética que no ha sido suficiente para enfrentar los dilemas éticos que viven en la realidad por ser eminentemente teórica y no aplicada a la práctica.

El enfermero no conoce y, por lo tanto, no ha aplicado la libertad de conciencia que le permitiría hacer objeciones de conciencia y de esta manera rechazar deberes que vayan en contra de su conciencia. A través del currículo se puede formar a la enfermera con una actitud éticay humanística.

\section{Bibliografía}

1. Angier N. La evolución de nuestra conciencia. En: El Tiempo. Santa Fe de Bogotá. 5 de mayo de 1997; 24-25.

2. Blondeau D. De l'éthique a la Bioéthique,gaetan, $2^{\mathrm{a}}$ ed. Quebec. Morin Ed.1986: 3-420.

3. Concilio Vaticano II. Constitución Gaudiun et Spes. No.16. 4. Corte Constitucional. Sala Tercera de Revisión. Sentencia T409 del 8 de Junio de 1992.

5. Fry ST. La ética en la Práctica de Enfermería. Consejo Internacional de Enfermeras. Ginebra. Suiza 1994.

6. Gamboa GA. La Objeción de Conciencia. Bioética. En: Boletín de Reflexión e Información. Academia de Bioética. Universidad del Valle. Cali. Abril de 1995.
7. Garzón N y otros. Fascículos de Etica. Asociación de Facultades de Enfermería Acofaen. Santa Fe de Bogotá. 1995.

8. Gasull Villeta M. Revista Rol de Enfermería. 1987; No 102.

9. González Alvarez LJ. Etica. Editorial el Búho. Santa Fe de Bogotá. 1998.

10. Hackenspield MM. Los derechos Humanos. La Práctica de Enfermería y la Práctica de los Profesionales de Salud. Revista Rol de Enfermería. No. 2. Santafé de Bogotá. 1995.

11. Madrid Malo M. Sobre las libertades de Conciencia y Religión. Serie textos de divulgación No.20. Defensoría del Pueblo.1993.

12. Madrid Malo M. Estudio sobre el derecho a la Objeción de conciencia. Serie textos de Divulgación No.7. Defensoría del Pueblo. 1994.

13. Madrid Malo M. Conferencia sobre Marco Jurídico y Filosófico de la Objeción de Conciencia. ESAP. Septiembre de 1997. 14. Madrid Malo M. La libertad de Rehusar. ESAP. Instituto de Derechos Humanos.1991.

15. Morales A, Guzmán R. La franja cultural como elemento del currículo oculto del Universitario Lasallista. Tesis de Grado. Universidad de la Salle. División de formación avanzada. Octubre de 1993.

16. Arroyo MP y otros. Etica y Legislación en Enfermería. McGraw Hill, Interamericana, $1^{a}$ edición, España. 1998.

17. Pinzón Contreras R. Conferencista. Seminario-Taller. Objeción de Conciencia. ESAP. 1997.

18. Romero Carlos María. La objeción de conciencia en la Práctica Médica. Cuadernos de Derecho Judicial.1997.

19. Sánchez Torres F. Temas de Etica Médica. Giro ed. Ltda. Santa Fe de Bogotá. 1995

20. Velez Correa LA. Etica Médica. ed. Cib. SantaFe de Bogotá. 1987.

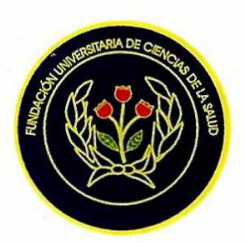

\title{
FUNDACIÓN UNIVERSITARIA DE CIENCIAS DE LA SALUD
}

HOSPITAL DE SAN JOSÉ

\author{
Personería jurídica $N^{\circ}$. 10917 Resolución M.E.N. $N^{\circ} .8125$
}

\section{PROGRAMAS DE POSTGRADO EN ENFERMERÍA}

PROGRAMAS

Cuidado intensivo

Neurología

Nefrología y Urología

Urgencias
CÓDIGOS

270256100791100111100

270256100721100111100

270256100771100111100

270236100731100111100
DURACIÓN

Tres semestres

Tres semestres

Tres semestres

Tres semestres

\section{PROGRAMAS DE POSTGRADOS INTERDISCIPLINARIOS}

PROGRAMAS

Gerencia en Salud

Gerontología
CÓDIGOS

270253526581100111100

270236100731100111100
DURACIÓN

Tres semestres

Tres semestres

Calle 10 No. 18-75 Teléfonos: 2018938 - 2019867 - 2015953 Telefax: 3513375 -2019561

E-mail: fucsalud@colomsat.net.co 